\title{
What is going on in the world? A display platform for media understanding
}

\author{
Dèlia Fernández*, Joan Espadaler*, David Varas*, Issey Masuda*, Aleix Colom*, David Rodríguez*, \\ David Vegas*, Miquel Montalvo*, Xavier Giró-i-Nieto**, Juan Carlos Riveiro* and Elisenda Bou* \\ *Vilynx, Palo Alto (CA) / Barcelona (Spain) \\ ** Universitat Politècnica de Catalunya, Barcelona (Spain)
}

\begin{abstract}
News broadcasters and on-line publishers daily generate a large amount of articles and videos describing events currently happening in the world. In this work, we present a system that automatically indexes videos from a library and links them to stories developing in the news. The user interface displays in an intuitive manner the links between videos and stories and allows navigation through related content by using associated tags.

This interface is a powerful industrial tool for publishers to index, retrieve and visualize their video content. It helps them identify which topics require more attention or retrieve related content that has already been published about the stories.
\end{abstract}

\section{Introduction}

In nowadays media, publishers as news broadcasters, magazines, bloggers, etc. are constantly generating large amount of video content and information. Automatically indexing all these contents, linking it to trends on social networks and events reported in the news is a task that can highly help identifying which topics are more relevant at a given time and detect contents that can be reused for publishing.

The contributions of our work can be summarized as a friendly user interface to display automatically indexed videos from a large on-line collection, relating it to trending stories on the news. Videos are updated to the system on real time through crawlers or RSS Feeds. On one hand, the system generates a multi-modal tagging of the videos using Knowledge Graph (KG) entities. In the other hand, it detects trending stories on the news and represents it as a set of relevant tags. Finally, the system detects events going

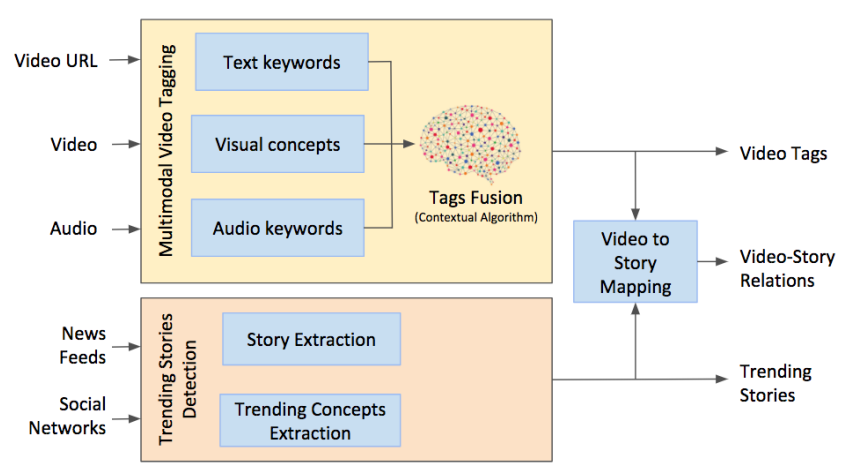

Figure 1. System pipeline scheme: A multimodal tags extractor and the trending stories detector. Information from both blocks is used to links videos to its related stories.

on in videos, and links it to trending stories. Currently this system is used by Vilynx customers, processing more than $30 \mathrm{k}$ stories per month and 50k video contents. The interface allows to navigate through related video content on the user video library in an intuitive manner. Moreover, it suggests possible trending videos based on its tags and related stories.

\section{System Overview}

In this section we give a brief explanation of the framework and pipeline that powers this demo. The system architecture is shown in Figure 1.

The videos are indexed with a rich collection of tags associated to $\mathrm{KG}$ entities. The system provides tags from three different sources: video, audio and associated text. The visual tagging algorithm provides detection on the persons appearing on the video. It is composed by a deep 


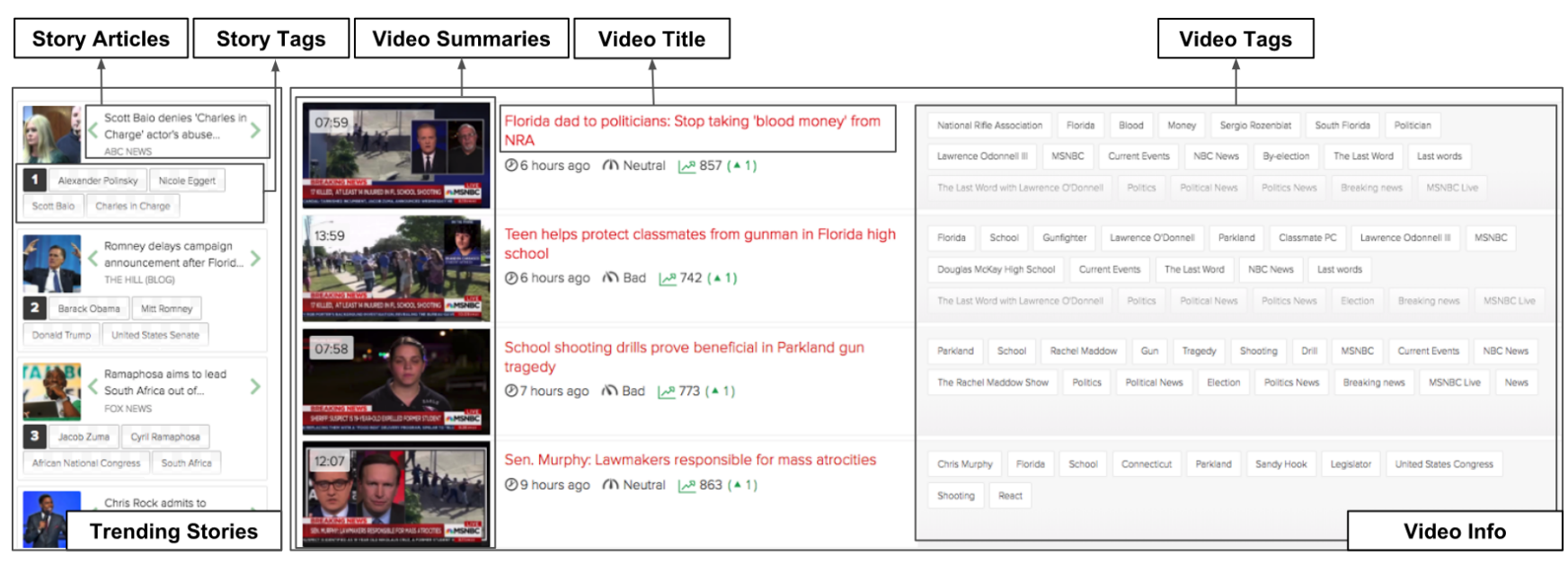

Figure 2. Platform main view consisting of a) trending videos at the center showcasing video summary, title and extracted tags and b) top trending stories on the left with its corresponding tags and related articles headlines.

learning model capable of recognizing over 9k different people. The tags from the text tagging block are generated by parsing the web page where the video is embedded and crawling the Internet in search of related content. Keywords are extracted from scrapped text through Natural Language Processing (NLP). After obtaining the audio transcript through speech to text algorithms, the audio keywords are extracted using NLP, as for the text keywords extraction. Finally, keywords and concepts extracted by the three multi-modal sources are mapped into KG entities or discarded in the Tags Fusion block. Due to space considerations, we have omitted the details of our system. For more information on the KG, the text tagging algorithm and the tags fusion see [1].

Each detected story is represented by a set of relevant tags describing it. Those tags are extracted from the related articles to the story topic. As videos are also defined by a group of tags, we link videos and stories using a metric that computes similarity between the tags in the video and the story.

\section{User Interface}

Our user interface displays contents from the video library and the top trending stories on the news. In Figure 2 we show the main page of the dashboard. On the left hand side we have the list of currently trending stories, ranked by a trending score. The most important tags associated with each story are displayed and also sorted according to the relevance score. Clicking on a story will take you to the individual story page, where we display the full list of articles that were identified as being related to the topic. We review the social impact of the story throughout the week in the Trending Chart, alongside examples of the story on social media. Lastly, when available, we aggregate all existing content about the story created by the individual publisher, as well as related content from their library.

\section{Conclusions}

We have presented a user interface to display video tags, trending stories on the news and its relations. It allows visualization and navigation between videos and related stories. This demo is an industrial platform that helps publishers order, visualize and further explode its video content through a friendly and intuitive dashboard.

\section{Acknowledgments}

Dèlia Fernàndez is funded by contract 2017-DI-011 of the Industrial Doctorate Programme of the Government of Catalonia. This work was partially supported by the Spanish Ministry of Economy and Competitivity under contracts TEC2013-43935-R and TEC2016-75976-R, and supported by grant 2014-SGR-1421 by the Government of Catalonia, and the European Regional Development Fund (ERDF).

\section{References}

[1] D. Fernández, D. Varas, J. Espadaler, I. Masuda, J. Ferreira, A. Woodward, D. Rodríguez, X. Giró Nieto, J. C. Riveiro, and E. Bou Balust. Vits: Video tagging system from massive web multimedia collections. In Proceedings of the 5th Workshop on Web-scale Vision and Social Media (VSM), pages 337346. IEEE Press, 2017. 